\title{
Design and Simulation of Cascade Fuzzy Control System for Boilers
}

Guo Kai

DongYing Technician College, College of Humanities, DongYing, China

\section{Email address:}

375540163@qq.com

\section{To cite this article:}

Guo Kai. Design and Simulation of Cascade Fuzzy Control System for Boilers. Science Discovery. Vol. 7, No. 6, 2019 , pp. 445-452. doi: 10.11648/j.sd.20190706.22

Received: November 25, 2019; Accepted: January 3, 2020; Published: January 4, 2020

\begin{abstract}
The control of boiler drum water level plays an important role in the automation control of boilers, the control effect directly affects the safety production of the boiler. In order to solve problems of non-linearity, instability and time-delay of boiler drum water level control system and difficulty in establishing accurate mathematical model, a feedforward-cascade three-flush boiler water level control system is designed, the Simulink control simulation system is established, and the control system is simulationed in the MATLAB environment, parameters of PID is determined according to engineering setting method, considering the PID controller exists some shortage,the adaptive fuzzy PID controller is designed and simulationed. The results of the system simulation analysis indicate, the three-pulse cascade and feedforward water level control system can effectively eliminate the load (steam amount) change and the disturbance of the feed water flow fluctuation, fuzzy adaptive PID has shorter response time and smaller overshoot than PID controller, to make the control water level meet the stable-operational requirement with high qualituy and accuracy, and can more satisfy the boiler drum water level control requirements.
\end{abstract}

Keywords: Fuzzy Controll, Drum Water Level, Three Impulses, Simulink, PID Controll

\section{锅炉串级模糊控制系统的设计与仿真}

\section{郭凯}

东营市技师学院人文学院, 东营, 中国

邮箱

375540163@qq.com

摘要：锅炉汽包水位的控制在锅炉的自动化控制中占据重要的地位, 其控制效果的好坏直接影响影响到锅炉的安全生 产。针对锅炉汽包水位控制系统具有非线性、不稳定性和时滞性及难以建立精确数学模型的问题, 设计了前馈-串级三 冲量锅炉水位控制系统, 建立了Simulink控制仿真系统, 并在MATLAB环境中对控制系统进行了模拟仿真, 并按照工 程整定的方法对PID控制器进行参数整定, 然后针对PID控制器存在的一些不足, 设计了模糊自适应PID控制器, 并对 该控制器进行仿真分析, 系统仿真分析的结果表明, 三冲量串级加前馈水位控制系统可以有效消除负荷（蒸汽量）变 化和给水流量波动的干扰, 模糊自适应PID具有比PID控制器更短的响应时间和更小的超调量, 能够快速使控制水位达 到稳定运行要求, 具有较高的调节质量和调节精度, 更能满足锅炉汽包水位控制的要求。

关键词：模糊控制, 汽包水位, 三冲量, Simulink, PID控制 


\section{1. 引言}

锅炉是化工、炼油、发电、发热等工业生产过程中必 不可少的重要的动力设备, 锅炉产生的高压水蒸气, 既可 作为风机、压缩机、大型泵类的驱动动力源, 又可作为蒸 馏、化学反应、干燥和蒸发等过程的热源 [1]。锅炉工作过 程中各项指标的调节难以建立数学模型, 具有非线性、不 稳定性、大滞后、大惯性的特点[2], 所以如何改善对锅炉 的控制并保证其正常工作, 提高工作效率一直是人们关注 的焦点[3]。

工业蒸汽锅炉汽包水位控制的任务是控制给水流 量使其与蒸发量保持动态平衡, 汽包水位是锅炉安全、 稳定运行的重要指标和重要参数, 即将锅炉水位控制在 合适的范围之内 [4], 由于控制对象在给水量扰动时有一 定的惯性, 在负荷扰动时又存在“虚假水位”, 采用传统 的PID控制方式很难适应，模糊控制能够有效适应锅炉 汽包水位控制系统工况,其不足在于控制精度低 [5]。模 糊 PID控制在锅炉温度控制中已经取得了较好的效果 [6], 通过MATLAB将DMC预测算法与前馈串级控制相 结合来提高锅炉水位控制系统的抗干扰能力, 取得了较 好的仿真效果 [7]。本文采用前馈-串级三冲量水位控制 系统能有效消除这些扰动, 以汽包水位为主信号, 任何 导致水位变化的扰动都会使调节器动作; 蒸汽流量作为 前馈信号, 防止 “虚假水位” 引起的调节器误动作, 改善 蒸汽流量扰动时的调节质量; 给水流量作为反馈信号, 使调节器能够在水位还没变化时就对前馈信号的变化 做出反应, 消除干扰[8]。

在建立锅炉汽包水位控制系统数学模型的基础上, 根据三冲量水位控制系统的原理 [9], 将前馈控制和串级 控制相结合, 设计了Simulink控制系统的仿真图, 对控 制参数进行分析和整定, 设计了自适应模糊PID控制器 提高控制性能, 并在MATLAB中对控制系统进行模拟仿 真。

\section{2. 汽包水位三冲量控制方式}

常见的锅炉汽包水位控制方式有单冲量控制、双冲量 控制和三冲量控制3种控制方式。单冲量水位控制系统是
水位控制系统中最简单的形式, 以汽包水位测量信号为唯 一的控制信号, 调节器根据汽包水位的偏差去控制调节阀, 以保持汽包水位在允许范围内, 单冲量控制方式只能应用 于控制要求不高的场合 $[10]$ 。

双冲量控制方式以蒸汽流量信号为前馈信号, 以水 位测量为控制信号, 当给水压力出现扰动时, 控制作用 相当于单冲量控制方式。三冲量控制方式引入了给水流 量信号, 采用蒸汽流量信号对给水流量进行前馈控制, 调节器能够迅速消除水压干扰引起的给水流量的改变, 能够对汽包水位进行控制, 有效的克服了“虚假水位”现 象 [11]。

通过分析发现, 相比于单、双冲量控制系统, 三冲量 控制系统具有控制效果好、可靠性高等优点, 能有效避免 “虚假水位”现象。

\section{3. 控制方案及数学模型的建立}

\section{1. 控制系统动态特性分析}

锅炉汽包水位控制的任务是维持汽包水位在工艺允 许的范围内。影响汽包水位变化的因素主要有燃煤量、给 水量、蒸汽流量、汽包压力等原因。燃煤量的影响非常缓 慢, 比较容易克服。因此, 给水量 $\mathrm{W} 、$ 蒸汽流量 $\mathrm{D}$ 是影响 水位的主要因素[12]。

\section{2. 前馈-串级三冲量控制方案}

锅炉水位控制系统的被控过程是具有大时延和大时 间常数的过程，串级控制在单回路控制的基础上增加一个 控制回路, 形成两个闭环, 副回路起着“粗调”的作用, 主 回路完成细调的任务, 控制效果和质量显著提高, 克服扰 动更为迅速有效, 且具有一定的自适应能力。前馈控制是 及时的、完全的补偿, 可以使被控参数不变或基本保持不 变, 对于时延常数或时延大、扰动大而频繁的过程有显著 效果[13], 因此我们将前馈控制和串级控制相结合, 组成 前馈-串级控制方式来提高控制质量。

前馈-串级三冲量控制框图如图1所示, 给水扰动作为 前馈信号, 加法器在控制器与调节器之间, 把给水扰动和 蒸汽扰动包括在副回路内, 以提高主参数的控制精度, 无 论如何设置系数, 当负荷变化时, 液位总能够保持无误差。

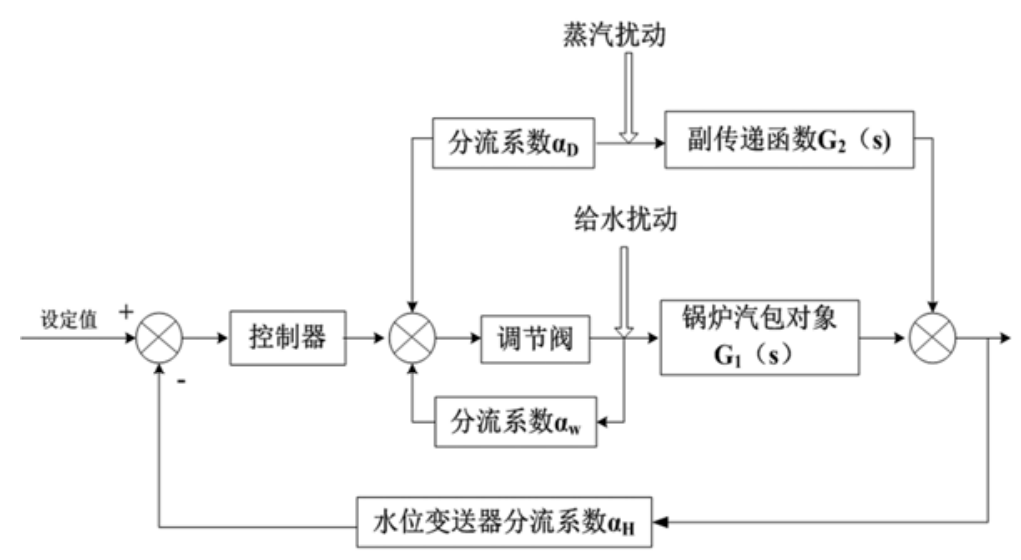

图1 前馈-串级锅炉控制系统。 


\section{3. 锅炉汽包水位控制系统的数学模型}

锅炉水位控制系统的传递函数及各参数值总体分为 三部分：在给水流量扰动下汽包水位的传递函数、在蒸汽 流量扰动下汽包水位的传递函数、各变送器的比例系数。

\subsection{1. 给水流量扰动下的汽包水位传递函数}

在给水流量发生阶跃响应时汽包水位的传递函数相当 于一个具有延迟时间的积分环节[14], 表达式及取值如下:

$$
G_{1}(s)=\frac{K_{1}}{s\left(T_{1} s+1\right)}=\frac{0.0529}{s(8.5 s+1)}
$$
常数。

$\mathrm{K}_{1}$ 表示水位的变化速度; $\mathrm{T}_{1}$ 表示水位的一阶滞后时间

\subsection{2. 蒸汽流量扰动下汽包水位的传递函数}

汽包水位的传递函数相当于一个积分环节和一个一 阶惯性环节，表达式及取值如下:

$$
G_{2}(s)=\frac{K_{2}}{T_{2} s+1}-\frac{\varepsilon}{s}=\frac{3.6}{15 s+1}-\frac{0.037}{s}
$$

$\mathrm{K}_{2}$ 为水位变化的放大倍数; $\mathrm{T}_{2}$ 为水位变化的时间常数, 即“虚假水位”引起的延迟时间; $\varepsilon$ 表示汽包水位对于蒸汽 流量的飞升速度。

\subsection{3. 变送器的比例系数}

假定液位变送器的量程为 $300 \mathrm{~mm}$ 水柱, 变送器标准信 号为 $0 \sim 10 \mathrm{~mA}$, 则水位变送器的比例系数:

$$
\alpha_{H}=\frac{10}{300}=0.0333
$$

给水流量和蒸汽流量变送器的比例系数为:

$$
\alpha_{\mathrm{D}}=\alpha_{\mathrm{w}}=\frac{10}{150}=0.0667
$$

\section{4. 汽包水位控制系统Simulink模型设计}

\section{1. 仿真模型建立}

由于MATLAB具有强大的矩阵运算能力和图形可视 化功能, 成为国内外首选的计算机工具[15], 本次仿真过 程涉及到高次微分方程的求解, 本文选用 MATLAB/SIMULINK软件对锅炉汽包水位控制系统进行 仿真。

主参数是系统操作要求的主要指标, 允许波动的范围 很小, 比例参数选择较大以保证系统稳定, 引入积分作用 增强控制作用, 因此主调节器采用PI控制规律, 采用试凑 法来确定PID控制器的参数, 最终确定的的PI参数为 $\mathrm{Kp}=9$, $\mathrm{K}_{\mathrm{i}}=0.0045$ 。副参数的设置起着快速调节作用, 允许有一定 的余差, 因此副调节器选择P控制规律。

结合前面所建立的数学模型及设计的前馈-串级控制 方案, 建立的Simulink仿真框图如图2所示, 为了更好的观 察扰动的影响, 将无控制器和PID控制器放在同一模型中 进行仿真。

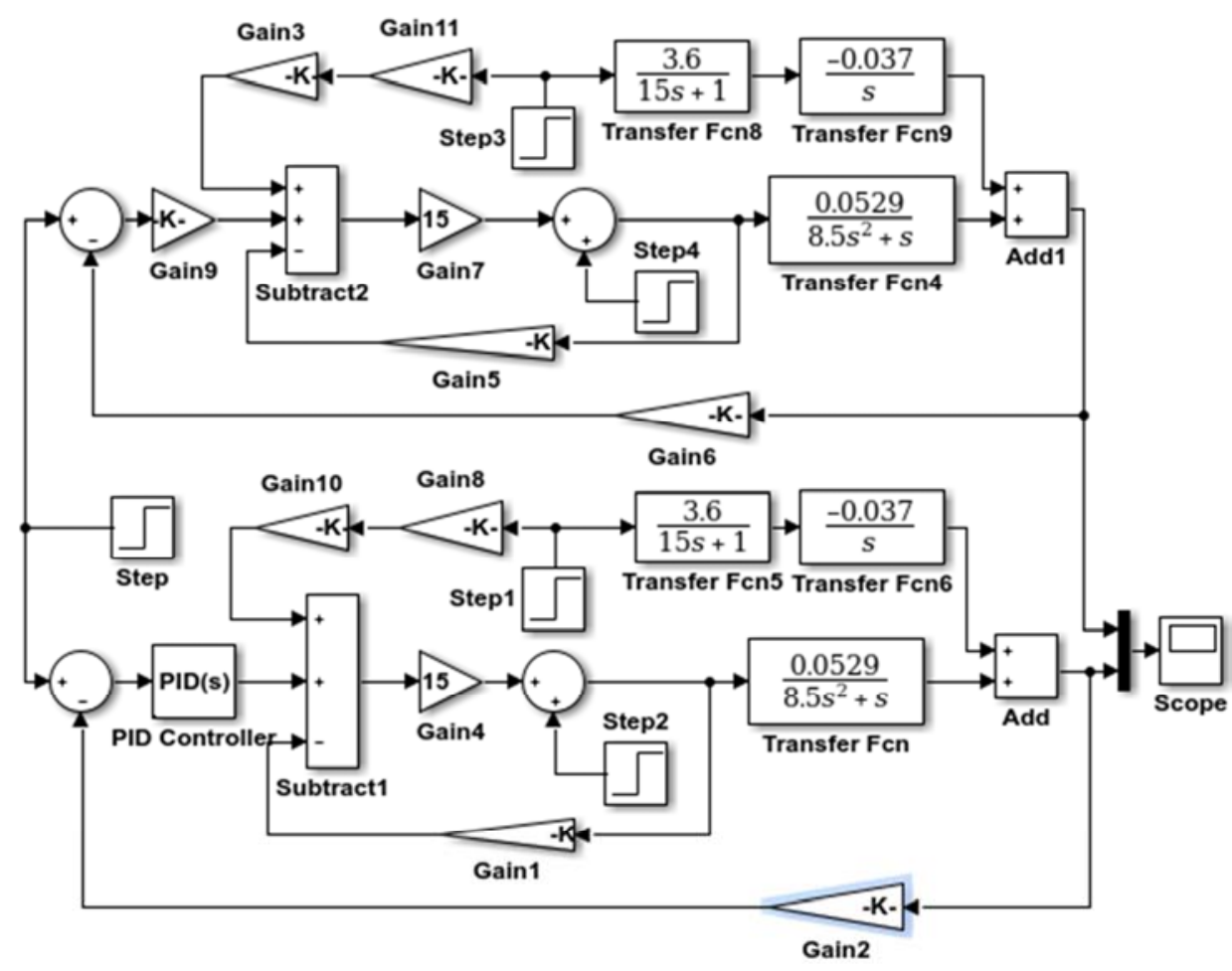

图2 锅炉汽包水位的三冲量串级PID控制系统。 


\section{2. 仿真结果分析}

仿真时, 阶跃函数设定值对应 $30 \mathrm{~mm}$ 水柱, 在 $200 \mathrm{~s}$ 同 时加入 $5 \%$ 的蒸汽扰动和 $5 \%$ 的给水扰动, 无控制器及PI控 制器在扰动下的系统闭环阶跃响应如图3所示。

从阶跃响应图中可看出: 当系统不加扰动时, 未加控 制器和PI控制器的前馈-串级控制系统基本在 100 s 处达到
稳定, 200s时加入扰动后, 无控制器的偏差明显增大, 而 对PI控制器的前馈-串级控制系统的阶跃响应几乎无影响, 采用PI控制器的前馈-串级控制系统可以克服给水扰动和 蒸汽扰动的影响。

从控制效果来看, PI控制的前馈-串级控制的系统响应 超调量较大, 响应时间较长, 须采用先进的控制方法对系 统进行校正。

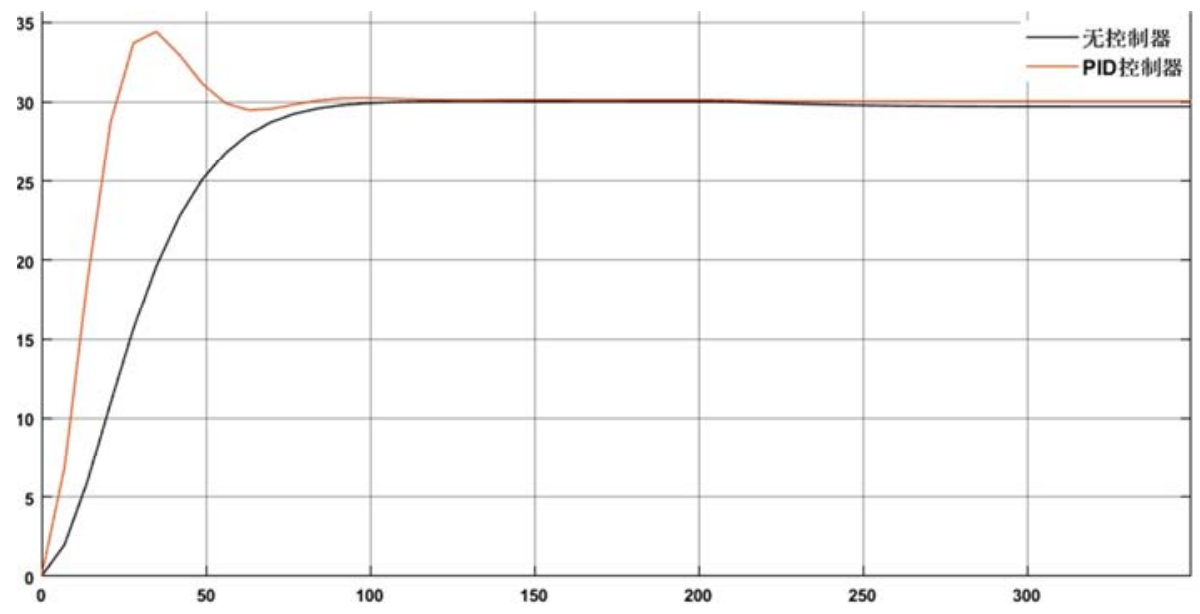

图3 加入蒸汽和给水量扰动时锅炉汽包水位仿真结果。

\section{5. 自适应模糊PID控制方案的设计}

常规PID控制器具有鲁棒性好和通用性强等优点, 但 由于参数固定，对于锅炉汽包水位控制系统来讲，参数一 旦整定, 对于系统中的不确定因素及环境的变化很难适应, 一组固定的PID参数也很难适应系统的全过程, 这样系统 的性能必然会受到影响, PID参数的重新人工调整也就意 味着运行成本的增加[16]。

针对上述控制系统中存在的上升时间、调节时间和稳定 时间较长的问题, 为满足系统的控制要求, 采用在线自调整
控制策略, 以其获得更好的控制效果。为此, 在常规PID的基 础上加上模糊自整定控制，根据系统响应偏差的特征，通过 Fuzzy推理实时在线调整PID的参数, 以达到更加满意的效果。

\section{1. 模糊自适应PID控制器结构原理}

由PID控制器和模糊控制构成的参数可调的模糊自适 应PID控制器结构如图所示, 以常规PID控制为基础, 模糊 控制以误差 $\mathrm{e}$ 和误差变化率 ec 作为二维模糊控制器的输入 变量, 以满足对控制器参数的不同要求。

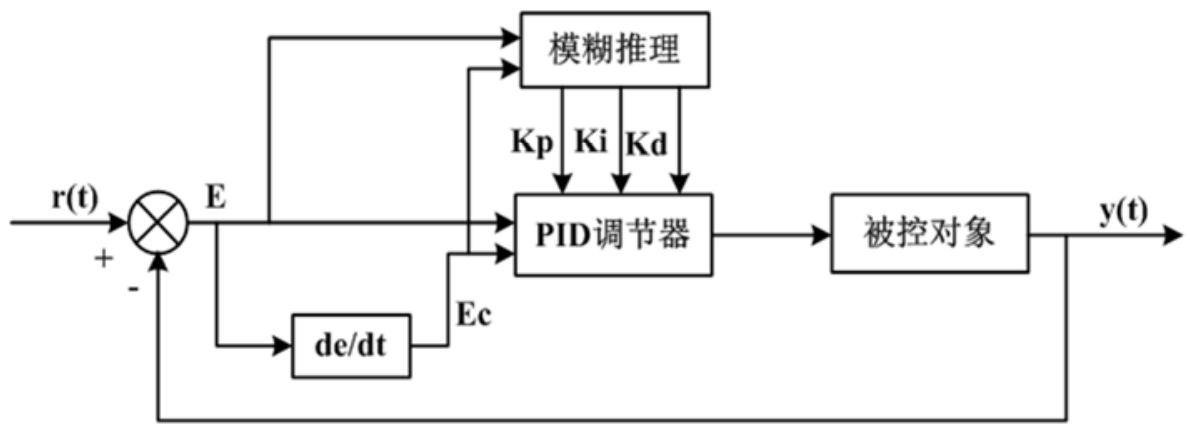

图4 自适应模糊控制器结构。

\section{2. 仿真模型的建立}

根据前面的分析, 系统用PI控制方式, 把误差e和误 差变化率 $\mathrm{e}_{\mathrm{c}}$ 作为输入, 控制器的两个参数 $\mathrm{P} 、$ I 的修正值 $\Delta K_{p}$ 、 $\Delta \mathrm{K}_{\mathrm{i}}$ 作为输出, 仿真模型如图5所示, 为了便于对比分析,
将PID控制器和自适应模糊PID控制器的仿真模型建立在 一起, 通过试凑法来确定自适应模糊PID控制器的各项参 数, 其中误差的量化因子 $\mathrm{K}_{\mathrm{e}}=110, \mathrm{~K}_{\mathrm{ec}}=80$, 经过模糊推理 后比例因子 $\mathrm{K}_{\mathrm{p}}=30 、 \mathrm{~K}_{\mathrm{i}}=0.2$, PI的初始值分别取 3.8 和 0.015 。 


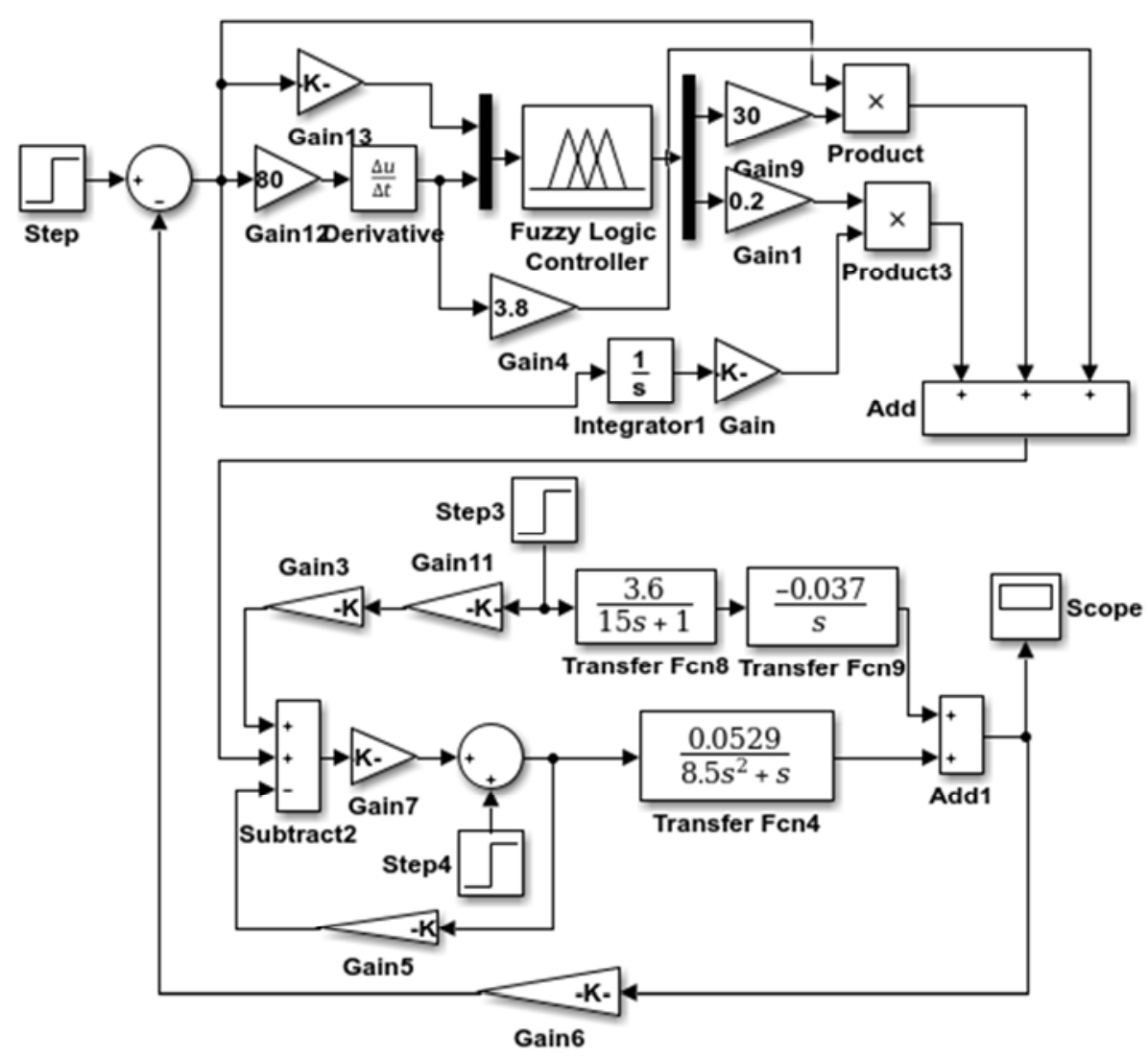

图5 自适应模糊PID锅炉水位控制系统。

5.3. 模糊论域及控制规则的建立

根据实际经验, 取e $e_{\mathrm{c}}$ 和 $\Delta \mathrm{K}_{\mathrm{p}} 、 \Delta \mathrm{K}_{\mathrm{i}}$ 的模糊子集为 $\{\mathrm{NB}$, $\mathrm{NM}, \mathrm{NS}, \mathrm{ZO}, \mathrm{PS}, \mathrm{PM}, \mathrm{PB}\}$, 基本论域和量化论域均
为 $[-6,6]$, 比例因子均为 1 , 三角形函数在论域范围内分 布均匀, 其灵敏度较高, 将其选作系统的隶属度函数, 如 图6所示。

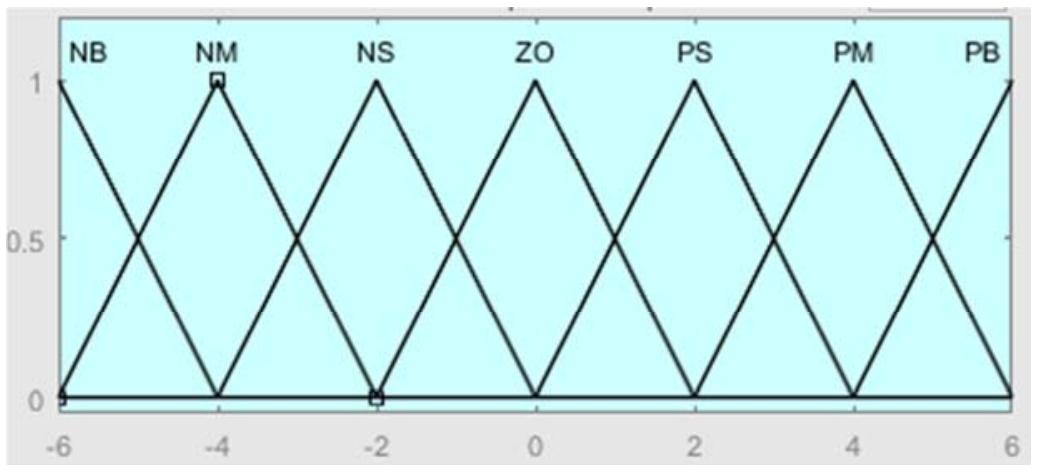

图6 e、ec和 $\Delta \mathrm{Kp} 、 \Delta \mathrm{Ki}$ 的隶属度函数。

在专家经验的基础上[17], 通过仿真经验进行调试, 归纳出PI参数的模糊控制规则表如表 $1 、 2$ 所示。

表1 参数 $K p$ 控制规则调整表。

\begin{tabular}{llllllll}
\hline ec & NB & NM & NS & ZO & PS & PM & PB \\
\hline NB & PB & PB & PM & PM & PS & ZO & ZO \\
NM & PB & PB & PM & PS & PS & ZO & NS \\
NS & PM & PM & PM & PS & ZO & NS & NS \\
ZO & PM & PM & PS & ZO & NS & NM & NM \\
PS & PS & PS & ZO & NS & NS & NM & NM \\
PM & PS & ZO & NS & NM & NM & NM & NB \\
PB & ZO & ZO & NM & NM & NM & NB & NB \\
\hline
\end{tabular}

表2 参数Ki控制规则调整表。

\begin{tabular}{llllllll}
\hline ec & NB & NM & NS & ZO & PS & PM & PB \\
\hline NB & NB & NB & NM & NS & NS & ZO & ZO \\
NM & NB & NB & NM & NS & NS & ZO & ZO \\
NS & NB & NM & NS & NS & ZO & PS & PS \\
ZO & NM & NM & NS & ZO & PS & PM & PM \\
PS & NM & NS & ZO & PS & PS & PM & PB \\
PM & ZO & ZO & PS & PS & PM & PB & PB \\
PB & ZO & ZO & PS & PM & PM & PB & PB \\
\hline
\end{tabular}




\section{4. 模糊推理系统的建立}

依据上述结果, 选择输入输出模糊变量的论域范围、 各语言变量的隶属函数形状等参数, 采用MAX-MIN规则
的模糊推理和重心解模糊化的方法来进行控制器的设计, 模糊推理系统中变量的定义和各变量隶属函数的设置完 成后, 界面如图7所示。

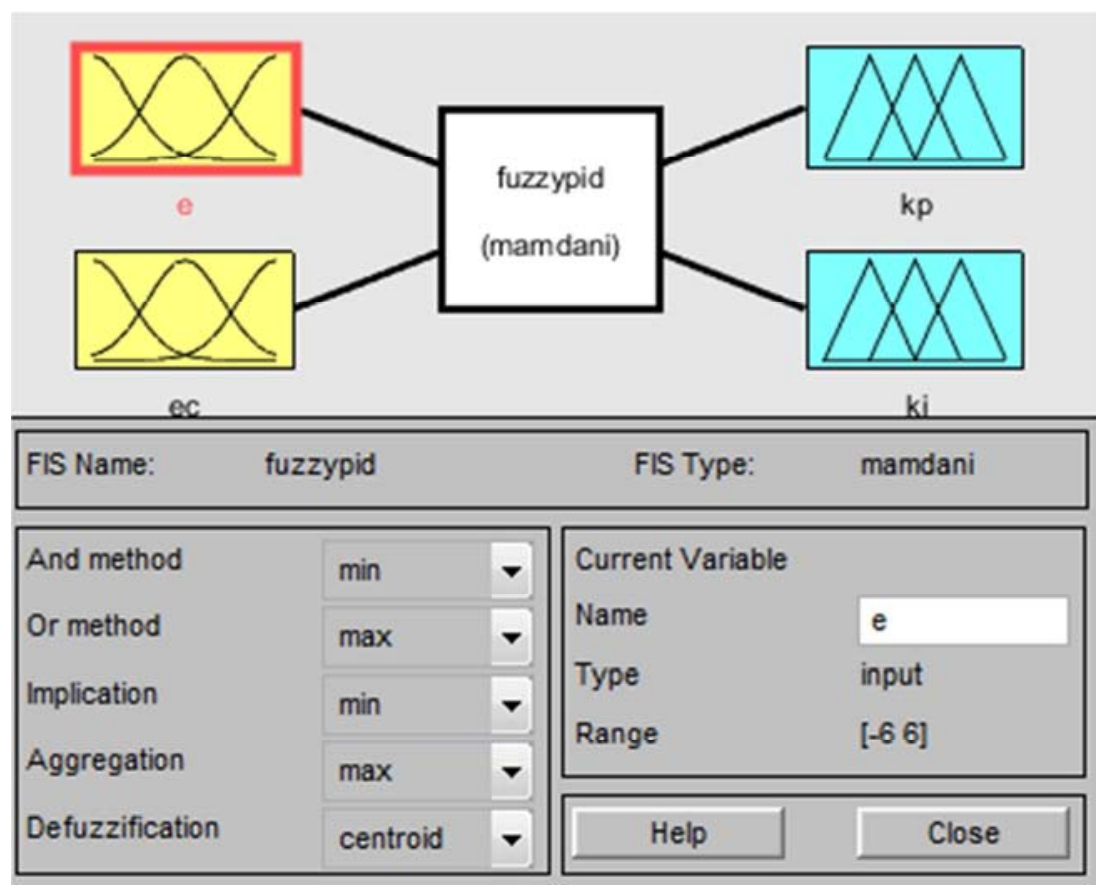

图7 模糊推理系统。

\section{5. 自适应模糊PID控制仿真及其比较研究}

仿真过程采用variable-step，采用ode-45的数字算法， 仿真时间取 $500 \mathrm{~s}$ ，在 $200 \mathrm{~s}$ 时分别加入 $5 \%$ 的蒸汽扰动和 $5 \%$
的给水扰动, 为了更好地显示自适应模糊PID控制的效果, 将其仿真模型同PID控制、无控制器控制放在一起, 仿真 后系统的阶跃响应曲线如图8所示。

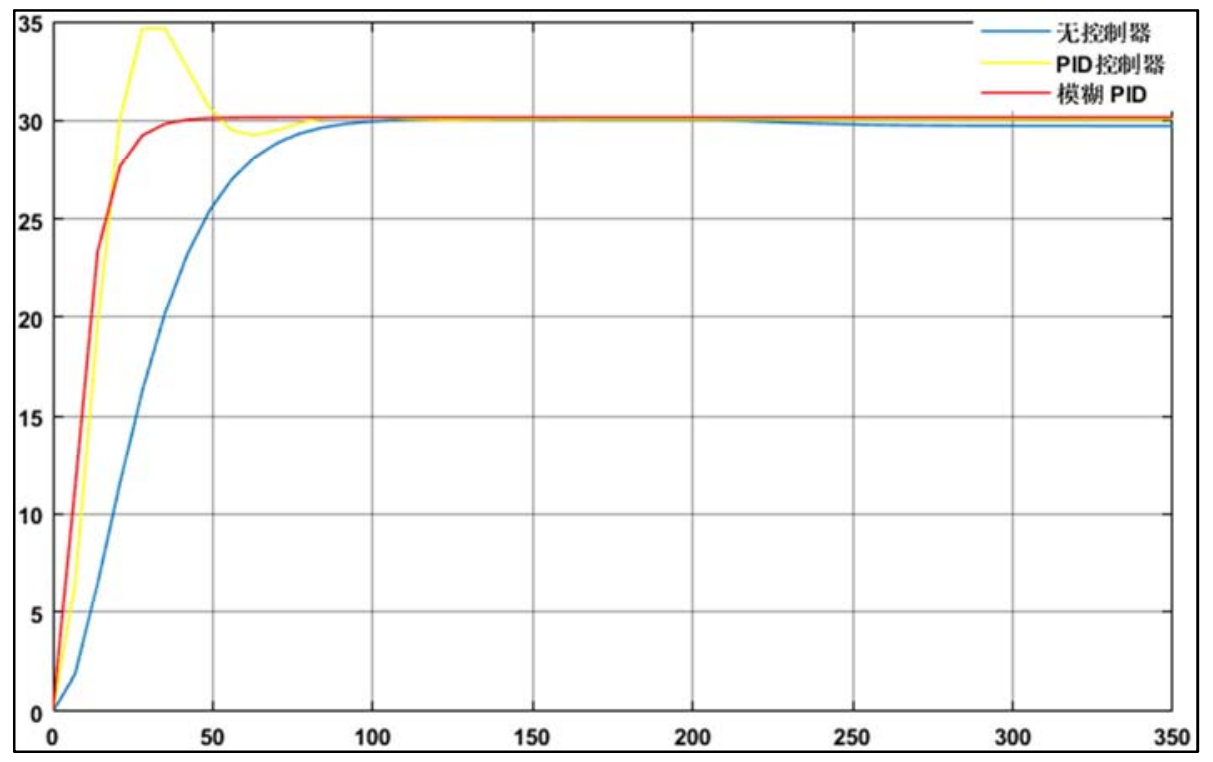

图8 蒸汽和给水量扰动下的锅炉液位控制系统仿真结果。

由仿真结果可以看出：自适应模糊PID控制虽然结构 复杂, 但控制效果的优势明显, 不仅克服了扰动的影响, 而且超调量较小, 上升时间短, 系统响应速度快, 控制精 度高, 充分体现了模糊PID参数自整定的效果。

\section{6. 结论}

在确定了锅炉汽包水位控制系统数学模型的基础上, 结合三冲量控制方式, 设计了前馈-串级控制方案来克服 
给水扰动和蒸汽扰动的影响, 在MATLAB/Simulink仿真平 台上建立了仿真模型, 通过仿真分析发现, PID控制的前 馈-串级控制相比无控制器控制能够克服扰动的影响, 但 存在系统的超调量较大, 响应速度较偏慢的问题, 为了克 服PID控制的缺点和不足, 将模糊控制和PID控制结合构成 了模糊自适应PID控制器, 可实现PID参数的在线动态调整, 通过仿真分析发现, 该控制方案不仅克服了扰动的影响, 在超调量、响应速度和控制精度方面, 模糊自适应PID控 制器表现出了更好的控制效果。

\section{参考文献}

[1] 张涛. 自动化专业毕业设计指导教程[M]. 北京: 煤炭工业 出版社, 2013:133-136。

[2] 张萍. 锅炉燃烧系统主蒸汽压力一给煤量的控制与优化 [D]. 陕西：西安理工大学, 2019: 20-24。

[3] 王震. PID控制技术在锅炉技术应用中的研究现状[J]. 民营 科技, 2016(4): 36-37。

[4] 白金金. 锅炉控制的智能优化算法研究 [D]. 河北: 河北科技 大学, 2019: 6-10。

[5] 黄斌, 王永树. 基于模糊PID的锅炉汽包水位控制系统研究 与应用[J].冶金能源,2019(3):56-58。

[6] 多晓艳. 工业锅炉智能控制系统的研究[D]. 河北: 河北科 技大学, 2019: 11-15。

[7] 刘涛, 张爽.基于DMC-PID算法的CFB锅炉水位控制系统仿 真研究 [J]. 现代工业经济和信息化, 2019(10):2225.
[8] 葛铮, 吴林峰. 基于模糊PID控制的锅炉主蒸汽温度控制策 略研究 [J].黑龙江电力,2018(4):358-361。

[9] Dongji Wang. Design and key heating power parameters of a newly developed household biomass briquette heating boiler [J]. Renewable Energy, 2019: 1371-1379。

[10] 张子才, 龚争理, 黄良沛. 锅炉汽包水位控制系统的设计 与仿真 [J]. 新技术新工艺, 2010(11):30-32。

[11] 廉迎战, 林德杰. 过程控制仪表及控制系统[M]. 北京: 机 械工业出版社, 2016:193-196。

[12] 乔金宇. 基于MATLAB/SIMULINK的锅炉汽包水位控制策 略及仿真分析[D]. 甘肃: 兰州理工大学, 2012:14-17。

[13] 窦艳艳, 钱蕾, 冯金龙. 基于Matlab的模糊PID控制系统设 计与仿真 $[\mathrm{J}]$. 电子科技, 2015 (2): 119-122。

[14] Li Yi-Min, Yue Yang. Adaptive Backstepping Fuzzy Control Based on Type-2 Fyzzy System [J]. Journal of Applied Mathematics ,2019 (5): 639-645。

[15] Shi Bin-Wan, Peng Xu. Estimation of distributed thermal boundary based on fuzzy clustering of temperature observable points[J].International Journal of Heat and Mass Transfer, 2019 (2): 335-339。

[16] 胡新新.基于自适应模糊控制的锅炉温度控制系统仿真分 析[J].中国自动化学会,2019:26-29。

[17] 刘金琨. 先进PID控制的Matlab仿真[M]. 北京: 电子工业出 版社, 2016: 283-287。 